\title{
La Enseñanza a Distancia Expande la Formación Académica e Integra a un Sector Técnico-Profesional al Trabajo en Acuicultura
}

\author{
(Distance-E ducation Extends Academic Learning and Integrate a \\ Technical-Professional sector to Aquaculture Work)
}

\author{
BEATRIZ GRACIELA BANNO \\ Universidad Abierta (Argentina) \\ J ADRANKA J URIC \\ Universidad Abierta (Argentina) \\ EDUARDO A.VALLARINO \\ Facultad de Ciencias Exactas y Naturales \\ Universidad Nacional de Mar de Plata (Argentina) \\ Juan Carlos Mallo \\ Facultad de Ciencias Exactas y Naturales - Investigador CIC \\ Universidad Nacional de Mar de Plata (Argentina)
}

\begin{abstract}
RESUMEN: El curso de extensión «Acuicultura: una propuesta para la generación de microemprendimientos» surgió en el año 1998, y continúa actualmente, de una acción coparticipada de la Facultad de Ciencias Exactas y Naturales y el área Universidad Abierta, ambas de la Universidad Nacional de Mar del Plata. Esta propuesta de alcancenacional resultó interesante para 163 estudiantes de todo el país. Estecurso es introductorio a la temática para personas con un nivel de educación inicial o medio y consta de 4 trabajos prácticos, 3 Actividades integradoras y 1 Anteproyecto final a desarrollarse durante 6 meses. Los alumnos fueron de todo el país, siendo de la región central $64,5 \%$ y de la región sur $28,2 \%$, del Noreste $(3,1 \%)$ y del Noroeste $(4,2 \%)$. En lo que respecta a los cultivos seleccionados se prefirieron en un $59 \%$ peces, $22 \%$ moluscos y en un $19 \%$ crustáceos. El $80 \%$ del total de los alumnos cursantes poséan una formación técnico-profesional, por lo que el curso otorgó una capacitación que cubrió demandas que no son abordadas por la enseñanza tradicional. Otro aspecto de la propuesta fue que tuvo mayor recepción en las zonas que ya poséan antecedentes en acuicultura (sur) y en donde la Universidad posee mayor presencia a través de su 20 centros regionales, en el centro del país (Bs. As.). La educación a distancia en esta temática dispone de una herramienta que puede propiciar el desarrollo denuevas áreas de producción y generar fuentes detrabajo para personas quehan salido del sistema educativo por temas laborales, de distancia o falta de interés.
\end{abstract}

acuicultura - maricultura - educación a distancia - formación continua - microemprendimientos 
ABSTRACT: The Extension course «Aquaculture: a proposal for the generation of little business enterprises» develops from 1998 to present days, as an inter-cooperation program between theFaculty of Natural and Exact Sciences and the Open University, both belonging to the Mar del Plata National University. This course was useful for 163 students of all the country, beig introductory to general aspects for people of initial or middle educational level. The course is based on 4 practical task, 3 integrity activities, and a preproject on aquaculture enterprise. Total duration is about 6 months. Students belong to different parts of the country, $64.5 \%$ of the Central region, $28.2 \%$ from the south, $4.4 \%$ of northwest and $3.1 \%$ of the northeast. Cultures of fishes were selected in $59 \%, 22 \%$ in mollusks, and $19 \%$ in Crustaceans. About $80 \%$ of students have a technical or professional formation. This allows qualification on aspects not included in traditional carriers. The course have more acceptance in traditional aquaculture zones, and also in the central region, due to the presence of about 20 educational university centers (Buenos Aires Province). Distance education is a good tool, that can be useful for development in new production areas, generating work for peoples out of the educational system due to work needs, distance to educational centers, and lack of interest.

Aquaculture - mariculture - distance education - continuous education - small business.

\section{INTRODUCGIÓN}

A partir de la acción coparticipada de la Facultad de Ciencias Exactas y $\mathrm{Na}$ turales y Universidad Abierta, ambas dependencias de la Universidad Nacional de Mar del Plata, se ha conformado una oferta de educación continua, donde la primera aporta sus docentes del Departamento de Ciencias Marinas, y la segunda los especialistas en Educación a distancia y semipresencial.

La Universidad propicia la capacitación continua en aspectos que ofrezcan soluciones concretas a problemas reales. El curso se ha diseñado con el propósito de capacitar recursos humanos en cultivos no tradicionales. Los proyectos de acuicultura en pequeña escala permiten generar ingresos además de desarrollar aspectos de pertenencia al lugar en el cual se habita. En una escala mayor, puede modificarse la estructura económica de la región, renovando actividades productivas, alimenticias, sociales y turísticas.

Los destinatarios son personas cuyo único requerimiento es el de contar con estudios secundarios. En esta primera etapa presentaremos el análisis del perfil del público destinatario, discriminándolo en diferentes variables, según las presunciones que se establecieron al momento de su diseño pedagógico. La correlación de algunas de ellas invita a establecer un análisis cualitativo muy interesante para el desarrollo de la educación continua con gestión a distancia.

\section{EL ENCUADRE INSTITUCIONAL}

La experiencia de extensión universitaria, con modalidad a distancia que vamos a presentar, surge a partir de la acción coparticipada de la Facultad de Ciencias Exactas y Naturales y el área de Universidad Abierta, ambas dependencias de la Universidad Nacional de Mar del Plata. 
La enseñanza a distancia expande la formación académica e integra a un sector Técnico-profesinal...

Para esta oferta de educación continua, se ha conformado a partir del año 1998, un equipo de producción, diseño y seguimiento de las intervenciones educativas conformado de la siguiente manera:

D Los docentes del Departamento de Ciencias Marinas, de la citada facultad, que cumplen el rol de contenidistas o especialistas en contenido.

- Los docentes de Universidad Abierta, especialistas en educación a distancia y semi presencial, que asumen el rol de procesadores pedagógicos y facilitadores del proceso de enseñanza y de aprendizaje. Completa el equipo de trabajo un área técnica y de apoyo, que cuenta con diseñador gráfico y personal administrativo. Para la edición y copiado de materiales impresos a la Imprenta de la Universidad, y para la asistencia informática al Centro de Cómputos.

La Universidad Nacional de Mar del Plata, de acuerdo con lo establecido en su Estatuto, propicia la capacitación continua en aspectos que ofrezcan soluciones concretas a problemas reales, resultado de demandas puntuales de la comunidad o producto de investigaciones situacionales. Es así que, en sus principios establece:

«...La Universidad deberá actuar como una de las herramientas de la sociedad con el fin de mejorar la calidad de vida de sus habitantes. Para esto, la Extensión Universitaria posibilitará una comunicación e interacción creadora entre la Universidad y la Comunidad.» (Art.22)

«La Universidad incentivará la realización de programas con proyección comunitaria que permitan la partici pación activa, organizada y eficaz de grupos interdisciplinarios constituidos por docentes, alumnos y /o graduados. »(Art.23).

«La función primordial de la Extensión Universitaria es la realimentación entre la Universidad y la Comunidad. Para ello, la Universidad deberá garantizar el proceso de transferencia académica e inserción curricular de los contenidos y/o metodologías extraídos del contacto con el medio.»(Art.24).

Si bien la Acuicultura es una actividad creciente; es posible consolidar su desarrollo planificando y coordinando tareas de capacitación y especialización, con el fin de asegurar su continuidad en el tiempo y en el espacio, minimizando la degradación del medioambiente. Para ello se hace indispensable contar con una adecuada información biológica, ecológica, socioeconómica y tecnológica.

A su vez, del análisis de la actual realidad económica y social argentina se desprende que se hace necesario encontrar alternativas que, tomando forma de emprendimientos, funcionen como células de crecimiento y desarrollo. El gran abismo de nuestro tiempo no es solamente entre pobres y ricos, sino también entre los que conocen y los que ignoran. De este abismo surgen peligros para la democracia que son similares a las tensiones sociales del pasado, por lo tanto en la formación moderna de adultos se trata no sólo, de preparar a los hombres para 
las exigencias siempre nuevas de su ámbito laboral sino también, las posibilidades de subsistencia en el mundo del mañana (Becker,1990).

\section{DESARROLLO DE LA TEMATICA EN LA ARGENTINA}

La acuicultura tiene una trayectoria de siglos en muchos países del mundo, fundamentalmente los asiáticos, siendo la herramienta por medio de la cual se han implementado micro y macro emprendimientos relacionados con la producción, alimentación, turismo y protección ambiental. En cualquiera de estos casos, son necesarios el conocimiento de las técnicas y de las características de las especies que pueden ser cultivadas, además de contar con la información de las tecnologías disponibles para cada tipo de explotación. A modo de referencia la acuicultura, como productora de alimentos ha incrementado su producción de manera impresionante. Entre 1975 y 1990 la producción mundial de alimentos por medio de la acuicultura fue de 13,1 millones de toneladas, entre 1990-1997, mientras que la pesca aumentaba un $9 \%$ la acuicultura llegaba a un $115 \%$, superando los 36 millones de toneladas. Estas cifras son aún más impresionantes si observamos que el principal productor mundial es China (67,8\% de la producción mundial de 1996), seguido por J apón, Noruega y USA (FAO, 1998).

Podríamos definir a esta actividad como de países de bajos ingresos y déficit de alimentos, pero con una fluida relación entre los centros de investigación, donde una adecuada extensión y transferencia a individuos resulta vital para el éxito de la producción.

La acuicultura provoca cambios en el medio ambiente, estos impactos se pueden analizar, fundamental mente según el manejo y elección del sistema de cultivo empleado. En general, la mayoría de las prácticas acuícolas han tenido poco efecto negativo en los ecosistemas. Sin embargo, se han registrado casos, en los cuales no hubo un manejo racional provocando la degradación del medio ambiente en zonas costeras. De allí que el impacto de estos emprendimientos sobre el medio ambiente apunta al denominado desarrollo sustentable (Bernabé, 1991).

En Argentina es incipiente su implementación destacándose el cultivo de salmónidos y pejerreyes en ambientes naturales como lagos, lagunas y embalses. Para un análisis más comprensivo del nivel de desarrollo de la producción acuícola en nuestro país, se toma en consideración su geografía y su clima y de acuerdo con ellos se divide en diferentes regiones (Fig.1):

I Región Noroeste que posee clima cálido y seco

II Región Mesopotámica, de clima cálido y húmedo

III Región Central, fundamentalmente de clima templado y con costa marina

IV Región Patagónica, con aguas frías de excel ente calidad y costas marinas. 
La enseñanza a distancia expande la formación académica e integra a un sector Técnico-profesinal...

Zonas de Acuicultura en la República Argentina

Figura 1: Zonas de cultivos acuáticos determinadas por la Secretaría de Agricultura, ganadería y pesca de la República Argentina. 1: Zona noroeste; 2: Mesopotamia;

3: Zona pampeana y 4: Patagónia.

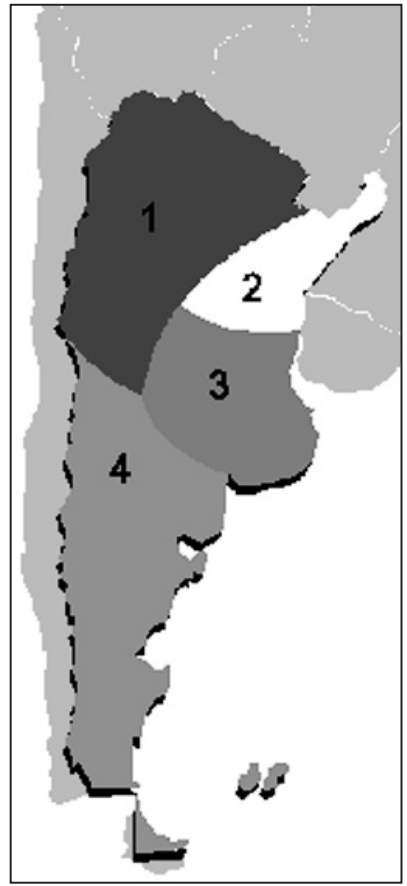

Figura 1

Con respecto a las especies que actualmente son utilizadas para el cultivo podemos mencionar en la región II, el camarón de agua dulce (Macrobrachium rosenbergi) o camarón gigante de la Malasia, con valores de producción de (año 1997) de 22,5 toneladas anuales. En la misma región y en forma de emprendimiento piloto se cultivan Tilapia (Oreochomis nilotica) y el Pacú (Piaractus mesopotamicus).

En cuanto a la maricultura en la región IV se desarrollan pequeños emprendimientos artesanales de mejillones (Mytilus edulis) en los Golfos Patagónicos y, se hallan en experimentación cultivos de ostras planas y ostras cóncavas. En escala piloto-comercial se desarrolla el cultivo de camarones peneidos como el langostino (Pleoticus mueleri) en la Región III y el camarón rosado (Peneus paulensis)

En una etapa experimental se están desarrollando en la región III cultivos de peces planos y truchas en lo que respecta a cultivos de agua de mar (en la provincia de Buenos Aires). 
Como panorama general, el desarrollo de la acuicultura es incipiente a pesar de las grandes posibilidades climáticas de la Argentina. Consideramos necesario un mayor desarrollo en los aspectos sociales que rodean a esta actividad sobre todo en zonas rurales donde los pequeños productores encuentren una alternativa a sus producciones tradicionales.

La acuicultura en la Argentina se ha desarrollado desde aproximadamente 20 años a esta parte sin grandes éxitos y fundamentalmente sustentada por esfuerzos particulares aunque en algunos campos se han obtenido logros significativos a nivel científico como en el cultivo experimental de ostras (Crassostrea gigas) según hemos podido comprobar en la mesa de debate sobre el presente de la maricultura argentina, en las ultimas J ornadas Nacionales de Ciencias del Mar, realizadas en el año 2000. Los pequeños avances se han dado con gran esfuerzo y sin un claro apoyo de promoción de parte del estado, lo que al presente dificulta el desarrollo de la actividad a escala piloto-experimental y comercial.

\section{EL DISEÑO DE LA OFERTA ACADEMICA}

La sociedad actual con un esquema de desarrollo económico regionalizado, donde la productividad y la competitividad incorporan como primer insumo recursos humanos con diferentes y variadas competencias de desempeños, va generando nuevos modelos de educación de adultos o de formación continua.

Cuando pensamos en el diseño de una oferta universitaria a distancia, nos inscribimos en la necesidad, reconocida en todos los sectores sociales, de una formación continua, ya sea desde sus programas de capacitación, actualización, especialización o desde la reconversión de sectores productivos y de servicios.

Al igual que en las nuevas vinculaciones sociales propuestas desde una nueva globalización y desde la incorporación de las nuevas tecnologías de la información y la comunicación, los sectores de producción establecen nuevas demandas, exigiendo la actualización y profesionalización de temáticas o desempeños que por su gran aplicabilidad deben instrumentarse con solvencia para que realmente den respuestas a problemas sociales.

El diseño curricular de un curso o programa es la forma concreta de accionar de una institución que realmente entra en contacto con el destinatario, de manera que:

- Se orienta con los propósitos institucionales, dando cuenta del tipo de re laciones de esta institución con la comunidad y responde a las condiciones contextuales particulares de los estudiantes.

De nutre de las preocupaciones clásicas de la didáctica: planteo de objetivos o expectativas de logro, los contenidos, las actividades, los supuestos 
La enseñanza a distancia expande la formación académica e integra a un sector Técnico-profesinal...

acerca del aprendizaje, los métodos y las estrategias metodológicas, que remiten a tiempos, espacios, selección de materiales y recursos tecnológicos, que mejor aseguren la generación y construcción del conocimiento a través de procesos cognitivos de distinto tipo, dentro de un determinado ambiente de aprendizaje

Al igual que el plan de estudios, el currículum designa un instrumento que sirve para facilitar el desarrollo planificado de la enseñanza, y además presupone una actitud dinámica, crítica, racional y constructiva frente a los fines y temas de la educación. En este sentido dice Omán (1990) que es concebi do como una aportación a la innovación democrática de la enseñanza a través de las instituciones públicas. Se puede entender su diseño como una exposición de los rendimientos en el aprendizaje y una prospección de las disposiciones del aprendiz para el aprendizaje.

Los modelos de diseño y desarrollo curricular vinculados a enfoques críticos consideran como un elemento fundamental los vínculos instituciones educativas/ sociedad que se manifiestan en el curriculum, dando al mismo una nueva dimensión de análisis de índole político ideológico. El análisis de las prácticas y la solución de problemas son uno de los ejes de lo curricular (Banno y del Río, 1998).

A partir de la síntesis que Stenhouse (1987) hace de varias acepciones de curriculum, podremos coincidir con él en que el diseño curricular presupone una tentativa para comunicar los principios y rasgos esenciales de un propósito educativo, de forma tal que permanezca abierto a discusión crítica y pueda ser trasladado efectivamente a la práctica.

El curso de Introducción a la Acuicultura se ha diseñado con el propósito de:

Capacitar recursos humanos para el desarrollo de cultivos no tradicionales

La estructura de logros propuesta es la siguiente:

D incorporar las premisas básicas para la instalación de la estructura, puesta en marcha y mantenimiento de los criaderos

D reconocer en cada especie su potencial como organismos de cría

D evaluar las condiciones de cría de las distintas especies

El diseño curricular del curso contiene los siguientes elementos estructurales:

D su iniciación con ejes temáticos permite visualizar los bloques conceptuales que conforman en sí mismos una unidad de aprendizaje.

D para una mayor explicación del camino de aprendizaje propuesto consignamos una estructura de logros, que describe al alumno las consolida- 
ciones consecutivas que deberá ir alcanzando, en aras de poder construir conceptos y procedimientos adecuados.

- para el abordaje de cada eje temático seleccionamos núcleos problemáticos o preguntas que nos sitúan en aquellos aspectos de mayor significación conceptual que iremos desglosando a través de las temáticas convergentes para que cada alumno pueda ir conformando su mejor respuesta.

Si bien toda la propuesta se estructura en un diseño, estos componentes no se constituyen en secuencias yuxtapuestas ni necesariamente secuenciales, sino que, cada uno de los momentos conforman nodos a partir del entramado que surge de las construcciones particulares que elabora cada participante. Estas construcciones dependen, fundamentalmente, de las experiencias previas y de las expectativas de logro propias, más allá de las planteadas en el diseño curricular. Las traducciones de estos procesos se consolidan en los proyectos particulares que el participante comienza a elaborar desde el comienzo del curso, en un proceso de construcción, complejidad y gradualidad creciente.. En dicho proceso, según Bleger (1964), el estudiante debería transformarse de receptor pasivo, en coautor de resultados, logrando que utilicen, que se "hagan cargo" de sus potencialidades como seres humanos.

- los tiempos son indicados en semanas, se establecen en relación con el grado de complejidad de cada unidad temática y están pensados en función de permitir a cada alumno la organización de su cronograma , de acuerdo con sus posibilidades reales de disponibilidad de horarios. En dicha disponibilidad él conjugará sus tiempos laborales, afectivo-sociales, de esparcimiento y de estudio.

D los trabajos prácticos, con sus correspondientes consignas, se encuentran incorporados en el Cuaderno de Trabajo, al finalizar cada eje temático y como integración teórico-práctica de los conceptos y procedimientos que han sido presentados. Las actividades integradoras, a modo de problemáticas, establecen otro tipo de resolución de problemas, constituyéndose en un momento de validación y contrastación de los conocimientos adquiridos.

- la evaluación en su instancia formativa está compuesta por los cinco Trabajos Prácticos, y dos Actividades de Integración. Todas ellas tienen retorno a Sede Central para su corrección y verificación de niveles de logros alcanzados. Completa los requisitos de acreditación un Trabajo Final que se solicita con formato de Anteproyecto, y en el cual se tienen en cuenta como insumos, los trabajos prácticos realizados a lo largo del curso, los cuales ya han merecido los comentarios y sugerencias de los tutores, y representaron para el alumno aproximaciones sucesivas al propósito del Curso.

Consideramos a la evaluación como parte del proceso didáctico. No como última etapa, ni como proceso permanente, sino instalada en el lugar mismo de la producción del conocimiento. El espacio que se genera permite al alumno la 
La enseñanza a distancia expande la formación académica e integra a un sector Técnico-profesinal...

reflexión sobre el propio aprendizaje, y al docente la posibilidad de acceder a la interpretación de los contextos de enseñanza:

«... las propuestas de construcción del conocimiento en las situaciones que lo posibilitan, dan cuenta de las situaciones de transferencia, permiten pensar bien los problemas del campo, tratan de resolver problemas reales y se constituyen, en la medida de lo posible, en procesos de resolución de problemas genuinos y consistentes con el campo de conocimiento eque se trate» (Litwin,1998).

\section{PUESTA EN MARCHA DEL CURSO}

El encuadre institucional sobre el que se comienza a trabajar el diseño del curso nos permite implementarlo como curso de EXTENSION UNIVERSITARIA. De esta manera confiábamos en poder abarcar sectores de población, no tradicionalmente universitarios. La expectativa era que se captara en la inscripción a un público que no necesariamente tuviera una vinculación académica anterior con el tema, sino que sus conocimientos previos fueran resultado de experiencias, intereses o expectativas relacionadas con las temáticas específicas o con la gestión de microempresas.

La primera cohorte se inició luego de un período de inscripción de dos meses, pero por las continuas demandas de información, se decidió incorporar las inscripciones y por ende los alumnos a medida que se recibían las mismas, generando cronogramas de entrega de trabajos de secuencia bimestral, lo cual complejizó todo el proceso de seguimiento del rendimiento de los alumnos y el apoyo de tutorías. Este criterio aproximó la oferta más a una concepción de educación abierta, adecuada al perfil, ritmos y demanda de los usuarios, que a sistemas formalizados. La implementación de la mencionada estrategia demandó un análisis aparte, por la forma de resolverse en la realidad.

En este trabajo presentaremos el análisis del perfil del público destinatario, discriminándolo en diferentes variables, según las presunciones que se establecie ron al momento de su diseño pedagógico. Los requisitos del perfil de entrada tipificaron a los destinatarios como personas cuyo requerimiento fuera el de contar con estudios secundarios, de manera no excluyente, atentos a que un curso con los propósitos que se habían planteado podría convocar usuarios con diferentes niveles de escolarización y de experiencias previas.

Teniendo como base las concepciones que como equipo de producción académico-pedagógico tenemos sobre el conocimiento, la enseñanza y el aprendizaje, los cuales constituyen, según Celman (1998), un marco referencial, epistemológico y didáctico, que juntamente con criterios ideológico-educativos y contextuales en que se desarrolla el proceso educativo, actúan a modo de parámetros para guiar nuestra reflexión y las interpretaciones, presentaremos los 
si guientes datos descriptivos sobre el ingreso producido entre los meses de marzo 1998 a junio de 2000.

Hemos considerado en la población solo a aquellos sujetos que habiéndose inscripto según los requerimientos del sistema de Universidad Abierta, habían tenido actuación académica. Entendiéndose por tal la presentación de uno o más de los trabajos solicitados. La distribución por sexo se observa como relativamente equilibrada (Tabla 1), porque, si bien hay una proporción mayor de participantes masculinos, podemos hablar de una cierta nivelación habida cuenta que esta actividad culturalmente se suele ubicar como masculina. Lo cual habla, creemos, de la inserción de la mujer en ámbitos de producción y explotación no tradicionales.

Distribución por género

\begin{tabular}{lcc} 
& $N^{\circ}$ de alumnos & $\%$ \\
Masculino & 58 & 60.4 \\
\hline Femenino & 38 & 39.5 \\
\hline Total & 96 & 100 \\
\hline
\end{tabular}

Tabla 1: Proporción de sexos entre los alumnos del curso Introducción a la acuicultura

La franja etaria entre los 18 y los 25 años estaría representando al grupo que tradicionalmente transita o desde la escuela media hacia estudios universitarios, o con estudios terciarios y universitarios incompletos. Una primera lectura permitiría inferir que no se sienten convocados por las carreras tradicionales y que habría una demanda significativa hacia estudios de nivel terciario. También podemos agregar que la población entre 18 y 45 años es la tradicionalmente atendida por los sistemas de educación a distancia. En nuestro caso representan más del $75 \%$ de los sujetos que se han matriculado (Tabla 2, 3 y 4).

Distribución etaria de alumnos

\begin{tabular}{cc} 
Franja etaria & Cantidad de participantes \\
\hline $18-29$ & $49(51,0 \%)$ \\
\hline $30-44$ & $26(28,1 \%)$ \\
\hline $45-59$ & $15(15,6 \%)$ \\
\hline+60 & $2(2,1 \%)$ \\
\hline No contesta & $4(4,2 \%)$ \\
\hline
\end{tabular}

Tabla 2: cantidad de participantes y porcentajes distribuidos por características etarias. 
La enseñanza a distancia expande la formación académica e integra a un sector Técnico-profesinal...

Nivel de estudios

\begin{tabular}{lccc} 
Estudios completos & Participantes & Área biológica & Otras áreas \\
Primario & $4(4 \%)$ & & \\
\hline Secundario & $54(56 \%)$ & & 6 \\
\hline Terciario & $12(13 \%)$ & $6(\mathrm{a})$ & 8 \\
\hline Universitario & $21(22 \%)$ & $15(\mathrm{~b})$ & \\
\hline No contesta & $5(5 \%)$ & & \\
\hline
\end{tabular}

Tabla 3: Distribución de los alumnos de acuerdo con el nivel de estudios en función de su relación con estudios biológicos.

(a) Prof. Ciencias Naturales (1), Técnico Superior en Acuicultura, (3), Técnico biólogo marino (1), Analista en Contaminación (1)

(b) Licenciados en Ciencias biológicas (5) , Prof. en Ciencias biológicas (1), Técnico en Acuicultura (1), Veterinario (3), Ingeniería química (1), Ingeniería agrónoma (2)

Población con estudios superiores incompletos

\begin{tabular}{lccc} 
& Participantes & Área biológica & Otras \\
Secundarios con estudios superiores incompletos & 35 & 26 (c) & 9 \\
\hline Terciarios con estudios superiores incompletos & 9 & 8 (d) & 1 \\
\hline Total $(\%)$ & 77,3 & 22,7 & \\
\hline
\end{tabular}

Tabla 4: proporción de alumnos con estudios superiores incompletos de áreas biológicas y de otras áreas.

(n) Técnico superior en Acuicultura (6), Lic. en ciencias biológicas (10), Ingeniería Pesquera (4), Veterinarios (3), Ingeniero agrónomo (1), Médico (1), Técnico Laboratorista (1)

(d) Licenciado en biología (2), Veterinario (2), Ingeniero agrónomo (1), Bioquímico (1), Ingeniería pesquera (1) Ingeniería en alimentos (1)

\section{ALGUNAS CONCLUSIONES SOBRE LO OBSERVADO}

1. Si bien es un curso de extensión universitaria, con requisito único y no excluyente de título secundario, el mayor impacto es en personas que tienen estudios superiores incompletos o completos en el área de las ciencias biológicas y derivadas.

2. En cuanto a la ubicación de los participantes en el curso, el mayor impacto se ha dado en la zona III, que es también coincidente con la zona de influencia de los 26 Centros Regionales de Universidad Abierta, a pesar de que es interesante también la captación de participantes de la zona IV, en la cual hay una reconocida cultura en la producción y explotación de productos acuícolas. 
3. En el cuadro de nivel de estudios (tabla 3), hay una distribución bastante equilibrada entre los alumnos con nivel secundario y los que se distribuyen entre el nivel terciario y universitario. Si cruzamos estos datos con los de estudios superiores incompletos en estos niveles y los orígenes de las formaciones profesionales de los participantes, se ve una interesante reinserción de alumnos con formación en el área biológica que ingresan nuevamente al sistema educativo para formación/perfeccionamiento/actualización en este campo específico. Si bien no contamos con datos sobre el tratamiento de esta temática en las formaciones de origen de los participantes, cabría preguntarse ¿Qué incidencia tiene esta área de conocimiento en las currículas específicas de la formación de grado de biólogos, licenciados en ciencias biológicas, profesorados, etc.? Su respuesta puede resultar un buen punto de inicio para recomendaciones de proyectos de grado en las Universidades Nacionales, asociadas a las estructuras de producción regionales.

4. Con respecto al grupo etario la mayor demanda se da en el grupo de edad entre 18 y 29 años. Una primera lectura permitiría inferir la falta de respuesta que las carreras terciarias están dando a formaciones profesionales no tradicionales, que tengan que ver con la producción y explotación de productos alimenticios.

Es evidente que la Universidad a través de sus programas de formación continua y a distancia, dispone de una herramienta que propicia el desarrollo de nuevas áreas de producción y la generación de fuentes laborales, en el marco de las microempresas para pequeños y medianos grupos, capacitando un sector de la población, que ha salido del sistema por deserción, abandono y que por su corte de edad se halla con otras obligaciones que no le permiten llegar a las aulas de manera tradicional. Creemos también (si se analizan los perfiles de los ingresantes) que sigue habiendo un reconocimiento, por parte de la sociedad, del papel de la Universidad como sistematizadora de conocimiento y como el ámbito idóneo de transferencia y profesionalización, captando ese papel también, para áreas no tradicionales y comprometidas con el desarrollo. Esto significa para la Universidad una mayor responsabilidad con ese supuesto y la necesidad de mantener ofertas académicas con dichas particularidades. 
La enseñanza a distancia expande la formación académica e integra a un sector Técnico-profesinal...

\section{REFERENGIAS BIBLIOGRÁFICAS}

BANno Y DEL Río (1998). El diseño curricular en la educación a distancia. Ponencia en XI J ornadas de Reflexión pedagógica. Universidad de Río Cuarto. Argentina

BeCKER, H. (1990) . La formación de adultos y su tarea. Tubingen. Alemania: Instituto de Colaboración Científica.

Bernabé, G. (1991). Acuicultura. Barcelona: Editorial Omega. a. Vol. 1 y 2

Bleger, J . (1964). Temas de Psicología., Buenos Aires: Ediciones Nueva Visión.

CAMILLONI, A. y COLB. (2000). Hacia un Sistema integrado de Educación Superior en la Argentina: democratización con calidad Secretaría de Educación Superior presentado en Coloquio Internacional: «La Educación Superior: Transformaciones y Tendencias»

CARR, W. y Kemmis, S. (1988). Teoría crítica de la enseñanza. Barcelona: Martínez Roca.

CARTIER, M.. (1992). Un nuevo modelo de acceso al conocimiento. En: Calidad, tecnología y globalización en la Educación Superior Latinoamericana. Venezuela: Caracas: UNESCO/ CRESALC.

Celman, S. (1998). ¿Es posibles mejorar la evaluación y transformarla en herramienta de conocimiento?. En La evaluación de los aprendizajes en el debate didáctico contemporáneo. Buenos Aires: Paidós Educador.

Cirigliano, G. (1983). La Educación Abierta. Buenos Aires El Ateneo.

CRESALC/UNESCO (1998). Plan deAcción para la Transformación dela Educación Superior en América Latina y el Caribe Caracas

Escotet, M. (1980) Tendencias de la Educación Superior a Distancia_. San José. Costa Rica. Universitaria.

F.A.O. (1998) Circular de pesca No. 815. Estadísticas de producción de acuicultura : 1987-1996

FILMUS, D. (comp.) (1998) Las transformaciones educativas en I beroameríca. Tres desafíos: De mocracia, desarrollo e integración. Buenos Aires. OEI y Editorial Troquel.

Gallart, M.A. y J ACINTO, C. (1997). Competencias laborales: tema clave en la articulación educación - trabajo En: M.A.Gallart y R.Bertoncello. Cuestiones actuales de formación. Montevideo:CINTEFOR,

Hohman, M. (1990) El curriculum y su construcción. Tubingen. Alemania: Instituto de Colaboración científica.

LiTwin, E. (1996). Tecnología educativa, Buenos Aires: Kapelusz,

LITWIN, E. (1998). La evaluación campo de controversias y paradojas o un nuevo lugar para la buena enseñanza. En: La evaluación de los aprendizajes en el debate didáctico contemporáneo. Buenos Aires: Paidós Educador.

LITWIN, E. (2000). La educación a distancia. Temas para el debateen una nueva agenda educativa. Buenos Aires: Amorrortu

RocA VILA, O. (1994). La autoformación y la formación a distancia: las tecnologías de la educación en los procesos de aprendizaje. En: Sancho, J. (coord.) Para una Tecnología Educativa, Barcelona, Horsori.

SILVIO, J . (1993). La comunicación del conocimiento en nuevo contexto tecnológico. En: Una nueva manera de comunicar el conocimiento. Venezuela: Unesco/Cresalc,

Stenhouse, L. (1987) . Investigación y desarrollo del currículo._Madrid: Ediciones Morata S.A., 


\section{PERFIL ACADÉMICO Y PROFESIONAL DE LOS AUTORES}

Beatriz Graciela Banno es Licenciada en Ciencias de la Educación. Realizó especialización en Educación a distancia (1985) en la UNMdP , con el Dr. Cirigliano; estudios de Maestría en Ciencias Sociales FLACSO (1994-1998), y formación de posgrado en el área de Teleformación, Universidad de Sevilla (2001).

Se desempeña como docente en Universidad Nacional de Mar del Plata, desde el año 1984, en las áreas de Educación a Distancia (1984-1987) y de Universidad Abierta (1987 a la fecha). Sus principales tareas de docencia corresponden a la constitución de equipos interdisciplinarios de trabajo para el diseño de propuestas y producción de materiales educativos y su implementación con modalidad semipresencial y a distancia. En carreras de grado ha diseñado asignaturas para Derecho, Bibliotecario Escolar, Tecnicatura en Administración Pública y Tecnicatura en Gestión Cultural. En el área de extensión universitaria ha trabajado con temas diversos, como por ejemplo la Prevención en el Uso Indebido de Drogas, Formación cooperativa, Acuicultura, etc. En docencia de posgrado se ha desempeñado como docente contenidista y tutora telemática en el curso «Bases conceptuales y modelos organizativos en educación a distancia», en sus ediciones 1999-2000. Su tarea de investigación se realiza en la Facultad de Psicología , Grupo de Psicología Cognitiva y Educacional, sobre el tema «Comunicación presencial y mediada por computadora. Procesos socio cognitivos comparados en ámbitos académicos».

bbanno@mdp.edu.ar

Eduardo A.Vallarino es Licenciado en Ciencias Biológicas, docente e investigador en la Universidad Nacional de Mar del Plata desde el año 1986. El tema de investigación es la aplicación de indicadores biológicos de contaminación en áreas marinas impactadas. Actualmente se está desarrollando esta línea de investigación en el grupo Bioindicadores Bentónicos de la Facultad de Ciencias Exactas y Naturales de la Universidad Nacional de Mar del Plata, con el financiamiento parcial de la empresa Municipal de Obras Sanitarias de la Municipalidad de Gral. Pueyrredón. Es autor de numerosos artículos de investigación en revistas nacionales e internacionales y artículos de extensión en el ámbito local. En cuanto a la tarea docente desarrolla su actividad en el ámbito universitario y como capacitador de docentes de distintos niveles educativos tanto municipales como provinciales. Desempeña también funciones docentes en Universidad Abierta como docente contenidista y como tutor en el curso «Acuicultura: una propuesta para la generación de microemprendimientos» ediciones 1999-2000-2001

evallari@mdp.edu.ar

J uan Carlos Mallo es Doctor en Ciencias naturales (UNLP) y Licenciado en Zoología (UNLP). Actualmente es Investigador de la Comisión de Investigaciones Científicas de la Provincia de Buenos Aires y de la UNMdP. Su proyecto de investigación actual es sobre Cultivo, fisiología y nutrición de crustáceos peneidos (UNMdP, CONICET). Es también Investigador Asesor «ad honorem» de la Subsecretaría de Pesca de la Provincia de Buenos Aires sobre desarrollo de cultivos no tradicionales. Ha realizado numerosas publicaciones en revistas nacionales e internacionales con referato y artículos de divulgación. Se desempeña como docente en la UNMdP en el período 1980-1983, en la UNS en los años 1984-1985, y nuevamente en la UNMdP desde el año 1992 a la fecha. Ha dictado varios cursos de perfeccionamiento sobre Acuicultura, Manejo de recursos acuáticos costeros, Biología marina, Contaminación y medio ambiente para la Dirección de Recursos Ictícolas y Acuícolas de la Secretaría de Recursos Naturales y Desarrollo Sustentable de la Nación, para la Dirección de E cología y Recursos Naturales de la Secretaría de Política Ambiental de la Provincia de Buenos Aires, para la Facultad de Ciencias Naturales y Museo de la Universidad Nacional de La Plata, para el Departamento Científico Zoología Vertebrados, para la Escuela Nacional de Pesca y para el Centro de Investigaciones Pesqueras en La Habana. Docente contenidista y tutor en el Curso «Acuicultura: una propuesta para la generación de microemprendimientos». Realizado conjuntamente con Universidad Abierta, con modalidad a distancia en sus ediciones de los años 1999-2000-2001

jcmallo@mdp.edu.ar 